Journal of Animal and Veterinary Advances 10 (8): 1073-1079, 2011

ISSN: $1680-5593$

(C) Medwell Journals, 2011

\title{
Exercise Effects on Menopause-Induced Changes in Heart of Ovariectomized Rats
}

\author{
Necip Fazil Kishali \\ Department of Physical Education and Sport, \\ School of Physical Education and Sport Sciences, Ataturk University, 25240 Erzurum, Turkey
}

\begin{abstract}
The menopause is characterized by the progressive decrease in estrogenic secretion and associated with bio-psycho and social changes which in turn impairs quality of life. This experiment was conducted to determine if exercise training has a protective role against the deleterious effects of aging in ovariectomized rats. Control rats and ovariectomized rats 12 weeks after surgery were subjected to a 4 weeks treadmill-running program. Subgroups established were young animals, old animals, ovariectomized animals, young exercisetrained animals, old exercise-trained animals and ovariectomized exercise-trained animals. In exercise groups, rats were subjected to treadmill exercise during which time each rat walked on a motor-driven treadmill at $15 \mathrm{~m} \mathrm{~min}^{-1}$ speed and $15^{\circ}$ incline once every 2 days during 10 days for three courses as 5,10 and $15 \mathrm{~min}^{-1 a y}{ }^{-1}$ totally for 30 days. In ovariectomized rat, heart tissue was remarkably dens fibrosis and an increase in necrotic cell density, possibly resulting from myocyte defect. Findings of old exercise-trained animal group more resembled young animal group rather than ovariectomized animal group and ovariectomized exercise-trained animal group.
\end{abstract}

Key words: Exercise, heart, menopause, myocyte, overiectomy, rats, Turkey

\section{INTRODUCTION}

The menopause is defined by the World Health Organization (WHO) as the point in time of permanent cessation of menstruation due to loss of ovarian function (Burger et al., 2002). A progressive decrease in estrogenic secretion during this period is associated to bio-psycho and social changes which in turn impair quality of life (Chedraui et al., 2009). Estrogen is a very important regulatory factor in all target structures of body which have two Estrogen Receptor (ER) subtypes denoted ER- $\alpha$ and ER- $\beta$. They are intracellular proteins that are members of a large superfamily of proteins and act as ligandactivated transcription factors (Katzenellenbogen et al., 2000). In estrogen deficiency as in menopause, execution of estrogen-mediated a lot of cellular or subcellular functions will not be possible in normal conditions to impair the regulation of ER- $\alpha$ and ER- $\beta$ expressions.

To be related to any kind of activity in the body with others directly or indirectly in this case, itself of many physiological processes regulated via estrogens are only be affected but also cause other negatives depending on the former. For example, menopausal period may be accompanied by many undesirable situations such as memory impairment (Dumas et al., 2010), depression (Dormænen et al., 2011), vasomotor and cardiovascular symptoms (Szmuilowicz and Manson, 2011) and increasing oxidative stress and metabolic disorders (Behr et al., 2011). Many questions remain to be answered are the hypothalamus and other brain centers also involved? How can we predict it. Why do some women suffer from these health problems more than others. Exactly how is the process of menopause involved in the increased risk for these health problems and conditions. Knowing these answers is likely to lead to better interventions and treatments that will delay, attenuate or even prevent altogether the decline in quality of life that accompanies menopause (Bellino and Wise, 2003).

When looking from another aspect how may be it affected other structure in the body such as heart, liver, kidney (etc. in terms of histopathology) are studies performed about menopause up to now enough for understanding it. Although, Cardiovascular Disease (CVD) constitution at any age, emerging evidence suggests that age and time since menopause deteriorate on cardiovascular outcomes. However, Manson et al. (2003) have reported that in clinical study coronary heart disease results were presented by time since menopause, the hazard ratios increased with greater distance from menopause (Manson et al., 2003). Also clinical studies showed that women are defended from CVD before menopause, suggesting an advantageous action of endogenous estrogens (Dubey et al., 2005). Addition of all these data on the vasculoprotective action of estrogens was also clearly demonstrated in all animal models of early atheroma (Clarkson and Appt, 2005; Holm et al., 1999). However, some beneficial effect of exogenous estrogens was inquired after the study of the heart and estrogen/progestin replacement study (Hulley et al., 1998) and the women's health initiative 
(Rossouw et al., 2002). These studies performed that postmenopausal women and comparing hormone replacement therapy versus placebo on major health outcomes (Adams et al., 1997). The initial results suggested overall harm from hormone replacement therapy, leading to a dramatic worldwide decrease in its use and concerns from clinicians and regulatory authorities. Today without any additional damage to the patients another important question for researchers is found in protection ways from possible negative effects of menopause which is impossible to be stop without hormonal therapy. Is one of solution ways physical exercise in addition to the other treatment protocols proven benefits. We know that there are close relationship between physical exercise and disease rate.

If menopause is accepted as a complex of abnormal conditions or a kind of disease and also estrogen deficiency is considered as an oxidative stress producing source, it is likely to try to connect a positive correlation between menopause and physical exercise. Also physical inactivity and ageing are widely showed appreciation of as risk factors for the result of developing of coronary artery disease. A female rat underwent ovx imitating a woman in the postmenopausal period. Therefore in this study, we tried to answer three questions:

- Does exercise training affect the in ovariectomized hearts?

- Can exercise training prevent changes in cardiac histological findings in ovariectomized rat hearts?

- Can exercise training have a protective role against the deleterious effects of aging?

\section{MATERIALS AND METHODS}

Animals and experimental groups: Forty-eight adult female Wistar albino rats, weighing 200-220 g were obtained from Ataturk University Experimental Animal Laboratory of Medicinal and Experimental Application and Research Center. Animal experiments and procedures were performed in accordance with the national guidelines for the use and care of laboratory animals and were approved by Ataturk University local animal care committee. Rats were housed in standard plastic cages on sawdust bedding in an air-conditioned room at $22 \pm 1^{\circ} \mathrm{C}$ under controlled lighting ( $14 \mathrm{~h}$ light $10 \mathrm{~h}^{-1}$ dark cycle). Standard rat chow and tap water were given ad libitum. The rats were randomly divided into 6 groups with each group containing eight rats:

- Young animal group (4-6 months)

- Old animal group (24-26 months)

- Ovariectomized animal group (9 months)

- Young exercise-trained animal group (4-6 months)

- Old exercise-trained animal group (24-26 months)

- Ovariectomized exercise-trained animal' group (9 months) (Table 1)

Ovariectomy surgery: Bilateral ovariectomy was performed as follows:

- Anesthetize rats with an intraperitoneal injection of $20 \mathrm{mg} \mathrm{kg}^{-1}$ thiopental sodium

- Shave the fur over the dorsal lumbar area

- Disinfect the skin with Betadine followed by an alcohol rinse

- A longitudinal incision $(0.5-1 \mathrm{~cm})$ was made in the midline area of the lower abdomen and the ovaries were removed

- Use cautery to control any bleeding

- Stitch the muscle with 3-0 absorbable sutures and use stainless steel wound clips (2-3 each side) to close the skin incision (Kharode et al., 2008; Albayrak et al., 2009)

After ovariectomy, rats were given $25 \mathrm{mg} \mathrm{kg}^{-1}$ metamizol sodium as analgesic for 2 days and $1.75 \mathrm{mg} \mathrm{kg}^{-1}$ amoxicillin once daily (i.m.) for 1 week to protect the host against infection.

Treadmill exercise: All exercise-trained rat groups were habituated to treadmill exercise during which time each rat walked on a motor-driven treadmill at $15 \mathrm{~m} \mathrm{~min}^{-1}$ speed and $15^{\circ}$ incline once every two days during 10 days for three courses as 5,10 and $15 \mathrm{~min}^{-1} \mathrm{yy}^{-1}$ totally for 30 days (Delp et al., 1993; Kwak et al., 2010; Choi et al., 2010).

\begin{tabular}{|c|c|c|c|c|}
\hline Groups & $\begin{array}{l}1-10 \text { days } \\
\text { (once evey other day) }\end{array}$ & $\begin{array}{l}11-20 \text { days } \\
\text { (once evey other day) }\end{array}$ & $\begin{array}{l}21-30 \text { days } \\
\text { (once evey other day) }\end{array}$ & 32 days \\
\hline Young (4-6 months old) & $*$ & $*$ & $*$ & Intaperitoneal thiopeltal \\
\hline Old (24-26 months old) & $*$ & $*$ & $*$ & Sodium $50 \mathrm{mg} \mathrm{kg}^{-1}$ \\
\hline Ovariectomized (9 months old) & $*$ & $*$ & $*$ & \\
\hline Young exercise-trained (4-6 months old) & $\begin{array}{l}15 \mathrm{~m} \mathrm{~min}^{-1} \text { speed and } \\
15^{\circ} \text { incline }+\end{array}$ & $\begin{array}{l}15 \mathrm{~m} / \mathrm{min} \text { speed and } \\
15^{\circ} \text { incline }+\end{array}$ & $\begin{array}{l}15 \mathrm{~m} / \mathrm{min} \text { speed and } \\
15^{\circ} \text { incline }\end{array}$ & \\
\hline Old exercise-trained (24-26 months old) & $5 \min$ day $^{-1}$ & $10 \mathrm{~min}$ day $^{-1}$ & $15 \min _{\text {day }}{ }^{-1}$ & \\
\hline
\end{tabular}

Ovariectomized exercise-trained ( 9 months old)

No exercise 
Tissue preparation processes: At the end of the experiment, the rats were killed by an overdose of a general anesthetic (thiopental sodium, $50 \mathrm{mg} \mathrm{kg}^{-1}$ ) and the heart samples were dissected out immediately and transferred into a $10 \%$ formaldehyde solution for light microscopy. On the following day, the samples were waited into same fixative (buffered formalin) for $24 \mathrm{~h}$ in room temperature.

After that routine preparation of samples according to conventional light microscopy they were dehydrated in graded alcohol series, embedded in paraffin wax and serially sectioned using a Leica RM2125RT microtome (Leica Microsystems, Wetzlar, Germany) and stained with Hematoxylin-Eosin ( $\mathrm{H}$ and $\mathrm{E}$ ) and Periodic AcidSchiff (PAS). All sections were studied and photographed by a light photomicroscope (Olympus $\mathrm{BH} 40$ ) (Altunkaynak et al., 2008; Halici et al., 2008).

\section{RESULTS}

\section{Conventional light microscopy by $H$ and $E$ and PAS} Young animal group (4-6 months): The three layers that constitute the heart wall, the endocardium, myocardium and epicardium were detected very well. The endocardium that constitute three distinct components called as a single layer of squamous endothelial cells, subendothelial layer, containing elastic and collagen fibers and smooth muscle cells and subendocardial layer including small blood vessels, nerves and Purkinje fibers were in normal appearances. The myocardium which is the muscular wall of the heart, attached the myocardium to the fibrous cardiac skeleton had no abnormalities.

Cardiac muscle cells, main component of the myocardium shown in normal features in myocytes such as a cross-striated banding pattern, centrally located palestaining nuclei, endomysial connective tissue containing a rich capillary network and. intercalated disks found at the interface between adjacent cardiac muscle cells Epicardium, the outermost layer of heart wall is also called visceral layer of the pericardium that is composed of a simple squamous epithelium known as a mesothelium were in normal appearances (Fig. 1a-f (group 1) and 2a-d (group 2)).

Old animal group (24-26 months): In old animal group, some of findings including such as protection structural integrity (Fig. 1a-f (group 1) and 2a-d (group 2)) were
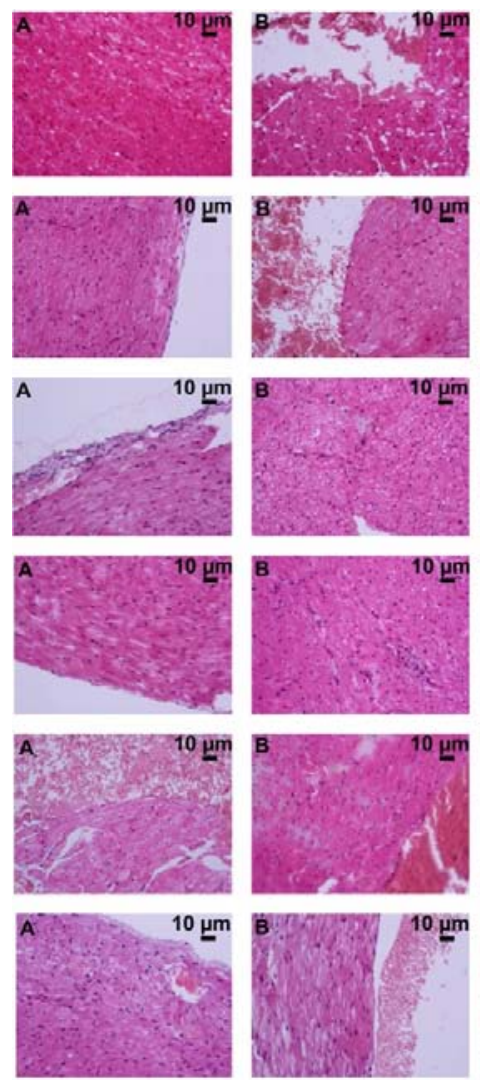
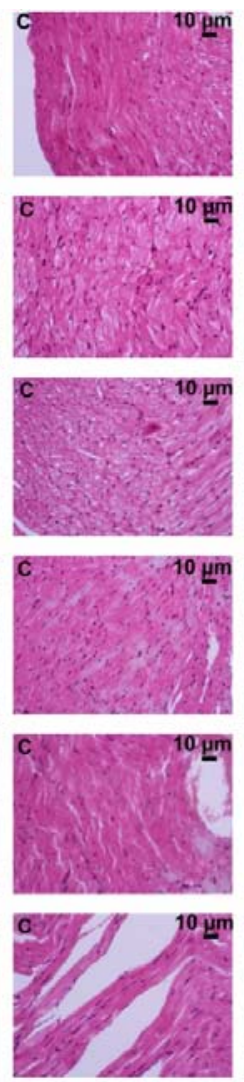
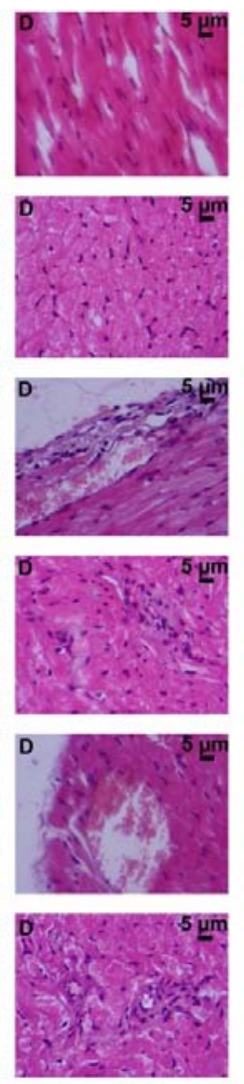
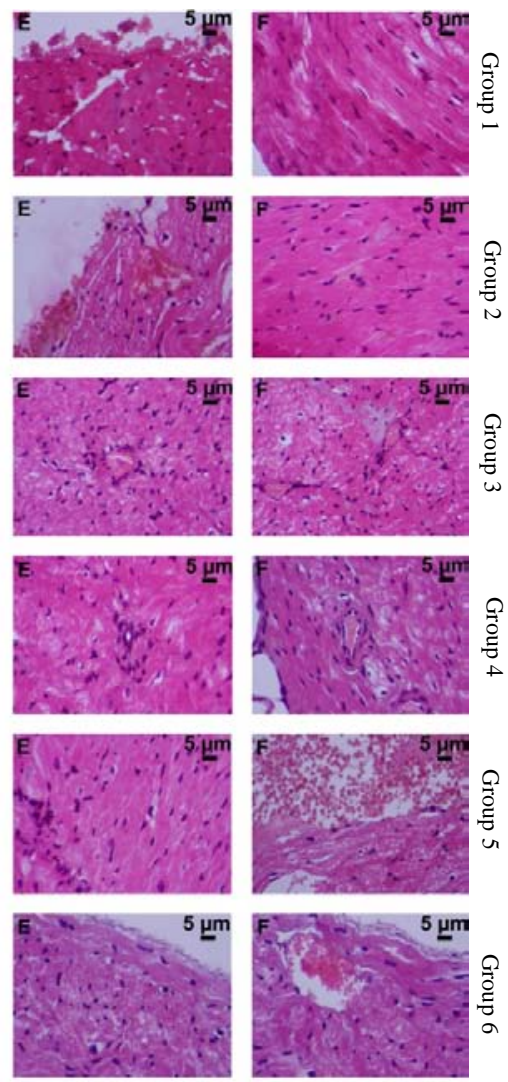

Fig. 1: Light microscopic photomicrograph of all groups (H and E) 

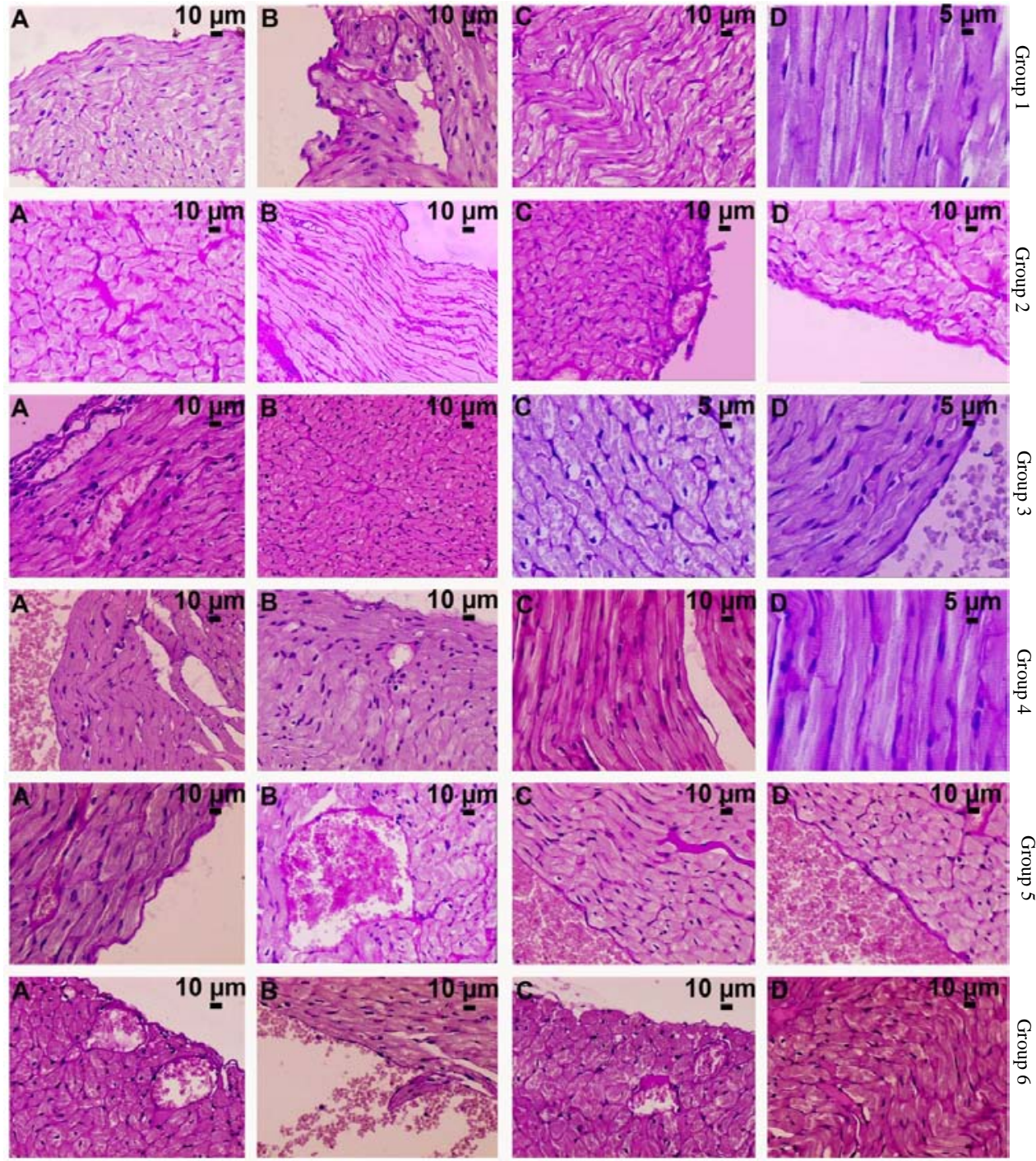

Fig. 2: Light microscopic photomicrograph of all groups (PAS)

similar to those seen in young animal group (Fig. 1a-f (group 1) and 2a-d (group 1)) and other findings such as an increase in fibrotic area and necrotic cell density and remarkable prominence in basement membrane thickness and amount of connective tissue elements were similar to those seen in ovariectomized animal group (Fig. 1a-f (group 3) and 2a-d (group 3)).

Ovariectomized animal' group (9 months): In ovariectomy groups as in old animal group there were similar findings, except for presence of very dens fibrosis and an increase in necrotic cell density as well as distribution of normal appearance of heart tissue (Fig. 1 a-f (group 3) and 2a-d (group 3)).
Young exercise-trained animal group (4-6 months): It was not observed significant change, all finding were similar to young animal group (Fig. 1a-f and 2a-d (group 4)).

Old exercise-trained animal group (24-26 months): It was determined a series of changes in the direction of improvement such as a decrease tendency in fibrotic area and necrotic cell density. It was also shown normalization in basement membrane thickness and amount of connective tissue elements (Fig. 1a-f and 2a-d (group 5)). Findings of old exercise-trained animal group (Fig. 1a-f and 2a-d (group 5)) more resembled young animal group (Fig. 1a-f and 2a-d (group 1)) rather than 
ovariectomized animal group (Fig. 1a-f and 2a-d (group 3)) and ovariectomized exercise-trained animal group (Fig. 1a-f and 2a-d (group 6)).

Ovariectomized exercise-trained animal group (9 months): Findings of this group were similar to old exercise-trained animal group (Fig. 1a-f and 2a-d (group 6)).

\section{DISCUSSION}

In this study, we evaluated some histopathological findings of myocardial structure in an in vivo menopausal rat model upon exercise training. Results from the present study in ovariectomized rats provided important and novel evidence for exercise training as a cardioprotective alternative in ovarian sex hormone-deficient condition. The study points to a possibility that regular running with moderate intensity could normalize the alteration in type of cardiomyocyte with ovariectomy. The initial results suggested overall harm from hormone replacement therapy, leading to a dramatic worldwide decrease in its use and concerns from clinicians and regulatory authorities. The study indicates the future important of exercise training as an additional alternative strategy. The American heart association scientific statement, recently has published the evidence for the efficacies of physical activity in the prevention and treatment of cardiovascular disease (Thompson et al., 2003). Now-a-days, there is evidence for confidence of increased exercise for the patients; additional physiological and basic study is needed to improve the scientific meaning to support the importance of such a recommendation. In the present study ovariectomy groups, other findings were almost similar to old animal group except two observations. One of them was very dens fibrosis and an increase in necrotic cell density.

Other was distribution of normal appearance of heart tissue. Finding of old exercise-trained animal group more resembled young animal group rather than ovariectomized animal group and ovariectomized exercise-trained animal group.

Scheuer (1982) discussed the hypothesis that physical exercises are beneficial to the heart also chronic repeated exercise increases myocardial vascularity and protects the myocardium against ischemic insult (Scheuer, 1982).

White et al. (1998) have reported that exercise induced cardiac capillary growth but this event rapidly followed by vascular remodeling which leads to an increase in the number and size of arterioles (White et al., 1998). Thus, reports on exercise-induced angiogenesis which focus on capillary density fail to convey the global picture of exercise-induced increase in cardiac blood supply.

Ho et al. (1983) presented that coronary vascular cast weights increased in relation to heart weight only after endurance rats were trained for 16 weeks by exercises and training (Ho et al., 1983). Treadmill training for long period increased the maximum coronary blood flow in dogs (Laughlin, 1985) and increased the number of coronary resistance arterioles in pigs (Breisch et al., 1986). Bronikowski et al. (2003) showed that exercise prevented from progress the effects of ageing on heart muscle by limiting changes in gene expression (Bronikowski et al., 2003).

There is now a critical mass of data to support that age or time since menopause importantly influences the benefit-risk ratio associated with heart histopathological changes, especially with respect to very dens fibrosis and an increase in necrotic cell density causes myocyte defect outcomes.

Clinically, the use of treadmill exercises would be too early to suggest for effective treatment of cardiovascular disease but at least exercise training as an additional treatment which is more common in late phase menopause. Additional research is needed to help postmenapausal women in decision making.

\section{CONCLUSION}

In this study, the use of treadmill exercises would be too early to suggest for effective treatment of cardiovascular disease but at least exercise training as an additional treatment which is already more common in late phase menopause

\section{REFERENCES}

Adams, M.R., T.C. Register, D.L. Golden, J.D. Wagner and J.K. Williams, 1997. Medroxyprogesterone acetate antagonizes inhibitory effects of conjugated equine estrogens on coronary artery atherosclerosis. Arterioscler Thromb Vasc Biol., 17: 217-221.

Albayrak, A., M.H. Uyanik, F. Odabasoglu, Z. Halici and A. Uyanik et al., 2009. The effects of diabetes and/or polymicrobial sepsis on the status of antioxidant enzymes and pro-inflammatory cytokines on Heart, Liver and Lung of ovariectomized rats. J. Surg. Res., (In Press). 
Altunkaynak, M.E., E. Ozbek, B.Z. Altunkaynak, I. Can, D. Unal and B. Unal, 2008. The effects of high-fat diet on the renal structure and morphometric parametric of kidneys in rats. J. Anat., 212: 845-852.

Behr, G.A., C.E. Schnorr and J.C.F. Moreira, 2011. Increased blood oxidative stress in experimental menopause rat model: The effects of vitamin A lowdose supplementation upon antioxidant status in bilateral ovariectomized rats. Fundamental Clin. Pharmacol., (In Press). 10.1111/j.1472-8206.2010. 00923.x

Bellino, F.L. and P.M. Wise, 2003. Nonhuman primate models of menopause workshop. Biol. Reprod., 68: 10-18.

Breisch, E.A., F.C. White, L.E. Nimmo, M.D. McKirnan and C.M. Bloor, 1986. Exercise-induced cardiac hypertrophy: A correlation of blood flow and microvasculature. J. Applied Physiol., 60: 1259-1267.

Bronikowski, A.M., P.A. Carter, T.J. Morgan, T. Jr. Garland and N. Ung et al., 2003. Lifelong voluntary exercise in the mouse prevents age-related alterations in gene expression in the heart. Physiol. Genomics, 12: 129-138.

Chedraui, P., G.S. Miguel and C. Avila, 2009. Quality of life impairment during the female menopausal transition is related to personal and partner factors. Gynecol. Endocrinol., 25: 130-135.

Choi, S.I., H.J. Chang, E.J. Chun, S.B. Cho and S.T. Kim et al., 2010. Exercise training improves agerelated myocardial metabolic derangement: Proton magnetic resonance spectroscopy study in the rat model. Korean Circ. J., 40: 454-458.

Clarkson, T.B. and S.E. Appt, 2005. Controversies about HRT-lessons from monkey models. Maturitas, 51: 64-74.

Delp, M.D., R.M. McAllister and M.H. Laughlin, 1993. Exercise training alters endothelium-dependent vasoreactivity of rat abdominal aorta. J. Applied Physiol., 75: 1354-1363.

Dormænen, A., M.R. Heimdal, A.C.E. Wang and A.S. Grimsgaard, 2011. Depression in postmenopause: A study on a subsample of the acupuncture on hot flushes among menopausal women (ACUFLASH) study. Menopause, (In Press),

Dubey, R.K., B. Imthurn, M. Barton and E.K. Jackson, 2005. Vascular consequences of menopause and hormone therapy: importance of timing of treatment and type of estrogen. Cardiovasc Res., 66: 295-306.
Dumas, J.A., B.C. McDonald, A.J. Saykin, T.W. McAllister, M.L. Hynes, J.D. West, and P.A. Newhouse, 2010. Cholinergic modulation of hippocampal activity during episodic memory encoding in postmenopausal women: A pilot study. Menopause, 17: 852-859.

Halici, Z., O.N. Keles, D. Unal, M. Albayrak and H. Suleyman et al., 2008. Chronically administered risperidone did not change the number of hepatocytes in rats: A stereological and histopathological study. Basic Clin. Pharmacol. Toxicol., 102: 426-432.

Ho, K.W., R.R. Roy, J.F. Taylor, W.W. Heusner and W.D. van Huss, 1983. Differential effects of running and weight-lifting on the rat coronary arterial tree. Med. Sci. Sports Exerc., 15: 472-477.

Holm, P., H.L. Andersen, M.R. Andersen, E. Erhardtsen and S. Stender, 1999. The direct antiatherogenic effect of estrogen is present, absent, or reversed, depending on the state of the arterial endothelium. A time course study in cholesterol-clamped rabbits. Circulation, 100: 1727-1733.

Hulley, S., D. Grady, T. Bush, C. Furberg, D. Herrington, B. Riggs and E. Vittinghoff, 1998. Randomized trial of estrogen plus progestin for secondary prevention of coronary heart disease in postmenopausal women. Heart and Estrogen/progestin Replacement Study (HERS) Research Group. J. Am. Med. Assoc., 280: 605-613.

Katzenellenbogen, B.S., I. Choi, R. Delage-Mourroux, T.R. Ediger and P.G.V. Martini et al., 2000. Molecular mechanisms of estrogen action: Selective ligands and receptor pharmacology. J. Steroid Biochem. Mol. Biol., 74: 279-285.

Kharode, Y.P., M.C. Sharp and P.V.N. Bodine, 2008. Utility of the ovariectomized rat as a model for human osteoporosis in drug discovery. Methods Mol. Biol., 455: 111-124.

Kwak, H.B., J.H. Kim, K. Joshi, A. Yeh, D.A. Martinez and J.M. Lawler, 2010. Exercise training reduces fibrosis and matrix metalloproteinase dysregulation in the aging rat heart. J. Federation Am. Soc. Exp. Biol., 25: 1106-1117.

Laughlin, M.H., 1985. Effects of exercise training on coronary transport capacity. J. Applied Physiol., 58: $468-476$.

Manson, J.E., J. Hsia, K.C. Johnson, J.E. Rossouw and A.R. Assaf et al., 2003. Estrogen plus progestin and the risk of coronary heart disease. New England J. Med., 349: 523-534. 
Rossouw, J.E., G.L. Anderson, R.L. Prentice, A.Z. LaCroix and C. Kooperberg et al., 2002. Risks and benefits of estrogen plus progestin in healthy postmenopausal women: Principal results From the Womens Health Initiative randomized controlled trial. J. Am. Med. Assoc., 288: 321-333.

Scheuer, J., 1982. Effects of physical training on myocardial vascularity and perfusion. Circulation, 66: 491-495.

Szmuilowicz, E.D. and J.E. Manson, 2011. Menopausal vasomotor symptoms and cardiovascular disease. Menopause, (In Press).
Thompson, P.D., D. Buchner, I.L. Pina, G.J. Balady and M.A. Williams et al., 2003. Exercise and physical activity in the prevention and treatment of atherosclerotic cardiovascular disease: A statement from the Council on Clinical Cardiology (Subcommittee on exercise, rehabilitation and prevention) and the Council on Nutrition, Physical Activity and Metabolism (Subcommittee on physical activity). Circulation, 107: 3109-3116.

White, F.C., C.M. Bloor, M.D. McKirnan and S.M. Carroll, 1998. Exercise training in swine promotes growth of arteriolar bed and capillary angiogenesis in heart. J. Applied Physiol., 85: 1160-1168. 